\title{
Understanding the role of $\mathrm{R} 266 \mathrm{~K}$ Mutation in Cystathionine $\beta$-synthase (CBS) Enzyme: An In Silico study
}

\author{
Aashish Bhatt and Md. Ehesan Ali* \\ Institute of Nano Science and Technology, Sector-81, Mohali, Punjab-140306 \\ India \\ *Email: ehesan.ali@inst.ac.in
}

\begin{abstract}
Human cystathionine $\beta$-synthase (hCBS) is a unique pyridoxal 5'-phosphate (PLP) dependent enzyme that catalyses the condensation reactions in the transsulfuration pathways. The specific role of Heme in the enzymatic activities has not yet been established, however, several experimental studies indicated the bi-directional communications between the Heme and PLP. Performing classical molecular dynamics (MD) simulations upon developing the necessary force field parameters for the cysteine and histidine bound hexa-coordinated Heme, we have investigated In Silico dynamical aspects of the bi-directional communications. Furthermore, we have investigated the comparative aspects of electron density overlap across the communicating pathways adopting the density functional theory (DFT) in conjunction with the hybrid exchange correlation functional for the $\mathrm{CSB}^{\mathrm{WT}}$ (wild-type) and $\mathrm{CBS}^{\mathrm{R} 266 \mathrm{~K}}$ (mutated) case. The atomistic dynamical simulations and subsequent explorations of the electronic structure not only confirm the reported observations but provide an in-depth mechanistic understating of how the non-covalent hydrogen bonding interactions with Cys52 control the such long-distance communication. Our study also provides a convincing answer to the reduced enzymatic activities in the R266K hCBS in comparison to the wildtype enzymes. We further realized that the difference in hydrogen-bonding patterns as well as salt-bridge interactions play the pivotal role in such long distant bi-directional communications.
\end{abstract}




\section{Introduction}

Homocysteine is a sulfur-containing amino acid that plays an important role in cell homeostasis. ${ }^{1}$ At the cellular level, a high concentration of homocysteine in the body causes severe myopia, atherosclerosis (cardiovascular disease and stroke), intellectual disability etc. ${ }^{2,3}$ This condition develops due to the deficiency of the Cystathionine beta-synthase (CBS) enzymes, which convert homocysteine to cystathionine in the transsulfuration pathway of the methionine cycle. CBS catalyses the condensation of homocysteine with serine (canonical activity) to form cystathionine in the transsulfuration pathway. ${ }^{4} \mathrm{CBS}$ also reacts with cysteine and is capable of producing $\mathrm{H}_{2} \mathrm{~S}^{5}$.-8. In higher organisms, CBS is the only PLP-dependent enzyme that has a regulatory heme-b cofactor. ${ }^{9,10}$ Structurally hCBS protein is a dimeric unit and has tendency to aggregate into oligomeric forms. ${ }^{10-12}$ Each subunit of hCBS is divided into three parts; N-terminal heme-binding domain, catalytic domain or PLP-binding site, and a Cterminal regulatory domain or S-adenosyl methionine (SAM) binding domain. In N-terminal domain (NTD), Heme is solvent-exposed and hexacoordinated with Cys52 $\mathrm{S}^{-}$(thiolate) and Nع2 atom of His65 at the axial positions. ${ }^{10-12}$ The exact role of heme is not yet well understood. However, Taoka et al. and Majtan et al. proposed the possible role of heme in the redox reaction as well as in protein folding. ${ }^{13,14}$ The absence of heme in the hCBS, the enzymatic activity was found to be reduced to around $20 \% .{ }^{15}$ This indicates the participation of Heme in the enzymatic reaction that occurs in the PLP centre in the catalytic domain. PLP is covalently bound to protein via internal aldimine Lys119 and it is the catalytic centre for the substrate reaction ${ }^{10-}$ 12,16. Several studies in the literature indicate the inhibition of CBS enzymatic activities when small molecules such as $\mathrm{NO} / \mathrm{CO}$ binds to Heme. The $\mathrm{CO}$ alter the Cys52 binding with heme and affect the Arg266 interaction whereas NO binding makes penta-coordinated system by attacking his65 site and eliminating binding with Cys52 also in hCBS. ${ }^{9}, 17,18,19$ The most striking fact is that the heme and PLP are located distant apart from each other by $\sim 20 \AA . .^{10-12}$

Based on the crystal structure of hCBS and several spectroscopic studies the bidirectional communication between the Heme and PLP sites has already been proposed to occur through $\alpha$-helix 8 that interacts at one end with the Cys52(S-) heme ligand via Arg266. ${ }^{10-}$ 12,17,20-24 (see Figure 1). On the other ends, conserved residue Thr-257 and Thr-260 residues interact with PLP and play an important role in the heme electronic environment and on activity. ${ }^{24}$ Smith et al. employing resonance Raman spectroscopy observed that the mutation in R266K invokes the geometric distortion into the heme due to Arg266-Cys52 interaction, that affects the PLP dependent activity(Fig 1). ${ }^{22}$ Therefore, it is inevitable to have an in-depth 
understanding on such long distant communication between the Heme that acts as electronreservoir being rich in quasi-degenerate pi-electrons as well d-electrons of the transition metal center, and the distal entity of PLP in which enzymatic reaction takes place.

A diverse functionality of the heme moiety in conjunction with different proteins have been explored using classical molecular dynamics simulation under different force field models. ${ }^{25-}$ 29 The unavailability of Amber force field parameters for cystine and histidine bound hexacoordinated heme present in hCBS calls for its parameterization under this specific chemical environment.

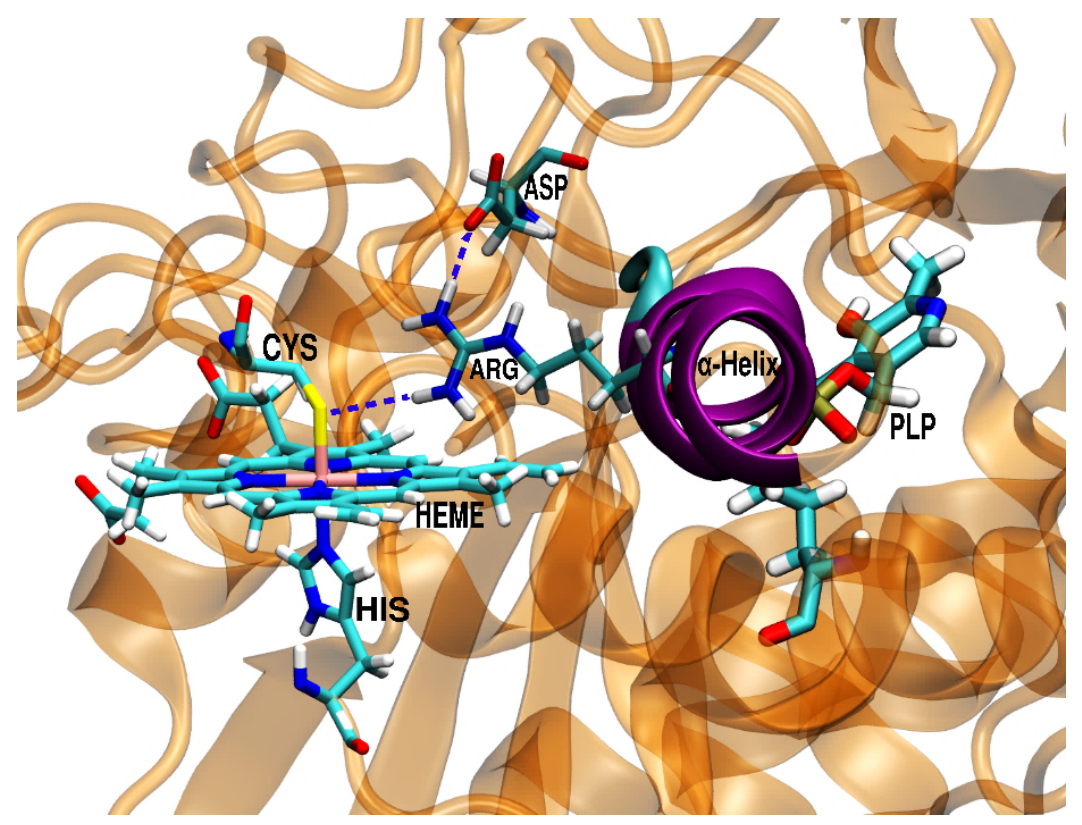

Figure 1. The cofactors and the key residues in hCBS enzyme that constitute the communication pathway between the Heme and PLP center. In the wildtype enzyme Arg266 forms strong non-covalent hydrogen-bond with the Cys52 as well as with the Asp316. Arg266 is covalently bound to alpha-helix 8 that further binds with the PLP that acts as the catalytic center.

Doss et al. have investigated structural and functional insight into the effect of SNP on the stability and activity of mutant CBS. ${ }^{30}$ In another work Gupta et al. studied the G307S substitution in the catalytic function and protein conformation. ${ }^{31}$ In this study, we have investigated the effect of R266K mutation in hCBS protein employing the atomistic molecular dynamics (MD) simulation as well as density functional theory. More specifically, here we intended to understand the structural dynamics and the effect of mutation on the local change in the hydrogen bonding and salt-bridge interaction patterns and their impact on the electron communication channel between the Heme and the catalytic center PLP. 


\section{Computational Methodology}

To perform the classical molecular dynamics simulations for human-Cystathionine betasynthase (h-CBS), the initial coordinates were obtained from the reported x-ray protein crystal structure (PDB ID 410D). ${ }^{11}$ The R266K mutated structure is generated by mutating residue using Chimera 1.13.1. ${ }^{32}$ The $\mathrm{pK}_{\mathrm{a}}$ values of the individual amino acids were calculated on PDB2PQR server. ${ }^{33}$ A python-based metal centre parameter builder (MCPB.py Builder) was utilized to build the force fields for Heme, which is connected to protein through the Histidine and Cysteine residue as the axis ligands. ${ }^{34}$

The force fields for such Heme are developed upon a rigorous benchmarking process. The details of force field generation and its benchmarking studies are provided in the supporting information. In brief, the molecular geometry of the Heme along with the axial ligands are optimized using three different hybrids functional (viz. B3LYP, M06 and PBE0) within the framework of Density Functional Theory (DFT). The magnitude for the force constant and the population analysis are performed using the optimized geometries. Antechamber in the AmberTools, are used to develop the PLP-Lys force field parameters (SI section 2).

The detail description for the computational methods adopted here to perform the MD simulation is provided in Sec.3 in SI. However, in brief, the protein with cofactor have been explicitly solvated in a $10.0 \AA$ margin TIP3P water ${ }^{35}$ box and $12 \mathrm{Na}^{+}$ions are added to the system to make it charge neutralized. The complete simulation system except the hexacoordinated heme has been parameterized with the amber ff14SB force field ${ }^{36,37}$ Particle mess Ewald method ${ }^{38}$ has been used to account for the long rage interaction with a cut-off distance of $12 \AA$. NAMD 2.13 package has been used to perform the classical MD simulations. ${ }^{39}$ Initially the system was equilibrated for $250 \mathrm{~ns}$. Additional $250 \mathrm{~ns}$ long trajectories were obtained for the production dynamics to obtain the statistical analysis. Visualisation and structural analysis are performed using VMD and Bio3 $\mathrm{d}^{40,41}$

\section{Results and discussion}

To understand the role and impact of R266K mutations in biochemical activities of hCBS enzymes, we have systematically compared the simulated MD trajectories with wildtype hCBS 
and analysed them applying a number of statistical and computational tools. In the following sections, the detail has been discussed.

\subsection{Comparison of the proteins' structure and dynamics}

To obtain an intriguing understanding on the protein dynamics, the RMSDs of the structural fluctuations are compared between the $\mathrm{CBS}^{\mathrm{WT}}$ and $\mathrm{CBS}^{\mathrm{R} 266 \mathrm{~K}}$. A comparison of the backbone atoms $(\mathrm{C} \alpha, \mathrm{C}, \mathrm{N}$, and $\mathrm{O}) \mathrm{RMSD}$ of the $\mathrm{CBS}$ protein are analysed Figure $2 \mathrm{a}$ and $2 \mathrm{~b}$. The RMSD of the entire protein and its decomposition along the multiple domains are given in Fig. 2 . The average RMSDs for the $\mathrm{CBS}^{\mathrm{WT}}$ and $\mathrm{CBS}^{\mathrm{R} 266 \mathrm{~K}}$ are found to be $3.38 \AA$ and $3.39 \AA$ respectively. The large fluctuations in the complete protein RMSD are primarily due to the intrinsically disordered regions of the N-terminal end that fluctuate larger than the other part of the protein. However, the average RMSD fluctuations of the catalytic domain for $\mathrm{CBS}^{\mathrm{WT}}$ is $1.30 \AA$ which is slightly larger than the mutated $\mathrm{CBS}^{\mathrm{R} 266 \mathrm{~K}}$ protein which is $1.26 \AA$. No significant difference is observed for the linker domains. It is also observed that the C-terminal domain (CTD) has larger fluctuations in $\mathrm{CBS}^{\mathrm{WT}}$ as compared to the $\mathrm{CBS}^{\mathrm{R} 266 \mathrm{~K}}$.

The root-mean-square fluctuations (RMSF) of the catalytic domain and all the residues are plotted in Fig. 2c. (also in SI S9) In the catalytic domain several residues, especially associated with R266K mutation (represented by a dotted line) shows significant differences in the RMS fluctuations. In this particular mutation, the protein structure exhibits a systematic lower RMSF fluctuation compared to the wild-type protein. This can be interpreted as a more compact protein structure for the mutated case. This might be a consequence of the smaller spatial volume occupied by the linear Lys266 as compared to the Arg266. A similar trend was also captured in the RMSD as well. Thus it indicates that a single mutation in the protein has a remarkable impact on the overall dynamics of the protein and also influence the atomistic motions of the other residues those are even quite far from the mutation sites. 



Figure 2. (A) RMSD calculation of $\mathrm{CBS}^{\mathrm{WT}}$ and $\mathrm{CBS}^{\mathrm{R} 266 \mathrm{~K}}$ with a different domain. Where residue Red is a complete protein (1-542 aa), Green is an N-terminal Domain (NTD) (1 to 70 aa), Blue is a Catalytic Domain(CD) (71 to 381 aa), Magenta is a linker (382 to 412 aa) and Brown is C-Terminal Domain(CTD) or regulatory domain (412 to 542 aa). (C) Catalytic domain comparisons of the RMSF for CBS ${ }^{\mathrm{WT}}$ and $\mathrm{CBSR}^{266 \mathrm{~K}}$.

\subsection{Dynamic Cross-correlation Matrix (DCCM) Analysis}

In the previous section, we discussed the residual fluctuation in $\mathrm{CBS}^{\mathrm{WT}}$ and $\mathrm{CBS}^{\mathrm{R} 266 \mathrm{~K}}$. For a deeper understanding of the correlated motion of the residues, we have computed all of the pairwise Pearson cross-correlation coefficients for the $\mathrm{C} \alpha$-atoms of all the residues. Crosscorrelation matrix (Fig. 3) provides a coarse and prompt picture of the most relevant linearly 
coupled motions occurring across the complete protein during the MD simulations.


Figure 3. Dynamic Cross-correlation maps were obtained as time-averaged for the CBS protein utilizing the complete simulation trajectory. The region of the black dotted circle indicates the region of the mutated site that has a strong positive correlation in the $\mathrm{CBS}^{\mathrm{WT}}$. Where cyan color bar belongs to the positive correlation, 1 (magenta) complete anti-correlation and 0 (White) there is no Correlation. Graphical represent Grey color (Nterminal), red (Catalytic domain), gap (linker), and green (C-terminal domain).

The dynamic cross-correlations analysis reveals that the atomic motions are strongly correlated in $\mathrm{CBS}^{\mathrm{WT}}$, though its RMSD and RMSF fluctuations are quite larger compared to the $\mathrm{CBS}^{\mathrm{R} 266 \mathrm{~K}}$. The comparison of the dynamical cross-correlation coefficients in Fig.3 indicates lesser correlated motion in $\mathrm{CBS}^{\mathrm{R} 266 \mathrm{~K}}$. We trace down this as the change in the non-covalent bonded interactions(see SI section 3.3). In the wild type, there is a very strong hydrogen bonding network with Cys52, Arg266, and Asp316 compared to the mutated case. We also find strong salt bridge interactions between the Asp316 and Lys266 compared with Arg266 and this could also contribute counter-intuitively to the correlations pattern.

\subsection{Long-range Communications between Heme and Catalytic Domain}

The allosteric communication between the Heme and the PLP catalytic center, which is almost $\sim 20 \AA$ apart can control the enzymatic activities. This has been established by Singh et al upon modulation of the electronic structure of Heme and investigated the PLP-dependent condensation reaction. ${ }^{17}$ To obtain atomistic and electronic details of such long-range communications the MD simulation trajectories are investigated in detail. It reveals quite a remarkable difference in the atomistic motions and electronic structures especially due to the 
R266K mutations. Arg266 interacts with the Heme through a strong hydrogen bond with Cys52. The probability distribution of the Cys52-Arg266 are shown in Fig. 4 and the maxima of the distributions are appeared at $2.28 \AA$ for the wild type hCBS. The singular peak confirms a stable and strong hydrogen-bond formation with rigid structural orientations of the Arg266 due to hydrogen-bonds with Cys52 as well as Asp316, This hydrogen-bond formation changes remarkably due to the R266K mutations. The probability distribution of Cys52(S-)--Lys266(H) distance along the MD trajectory (Figure 4) results in a broader distribution with the appearance of two peaks at $2.81 \AA$ and $3.38 \AA$. This indicates the hydrogen-bond formation in the mutated case is quite weak (or more precisely "less probable") compared to the wild type hCBS.-This is the primary reason behind the decrease of allosteric communications between the Heme and the catalytic PLP center due to the R266K mutations.



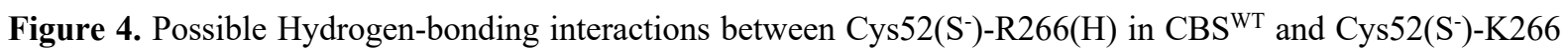
in $\mathrm{CBS}^{\mathrm{R} 266 \mathrm{~K}}$ are depicted in the upper panel (a) and (b) respectively. The probability distributions of these hydrogen-bonds along with the distance between the Cys52(S-)-R/K266(N) are shown in the bottom panel (c) and (d) respectively. The dual peaks for the mutated case in (c) indicate the hydrogen atoms responsible for these interactions (in (b)) with the Heme are in continuous rotational motions.

To get a deeper understanding about the non-bonding interactions we have further investigated the electron density distribution computed with PBE0/6-31G* within the hybrid 
density functional theory (DFT) framework. We have adopted a few distinct snapshots from all over the distribution (Figure 5). It is quite evident that for the wild type hCBS, at the most probable distance $(\sim 2.28 \AA)$, there is a strong overlap in the electron density between the interacting residues; Cys52 and Arg266. However, no such overlap in the electron density is observed at the most probable configuration for the R266K mutated case (Figure 5). Thus, it is quite evident that the R266K mutations not only break the strong hydrogen-bonding pattern but also modulate the electronic communications.

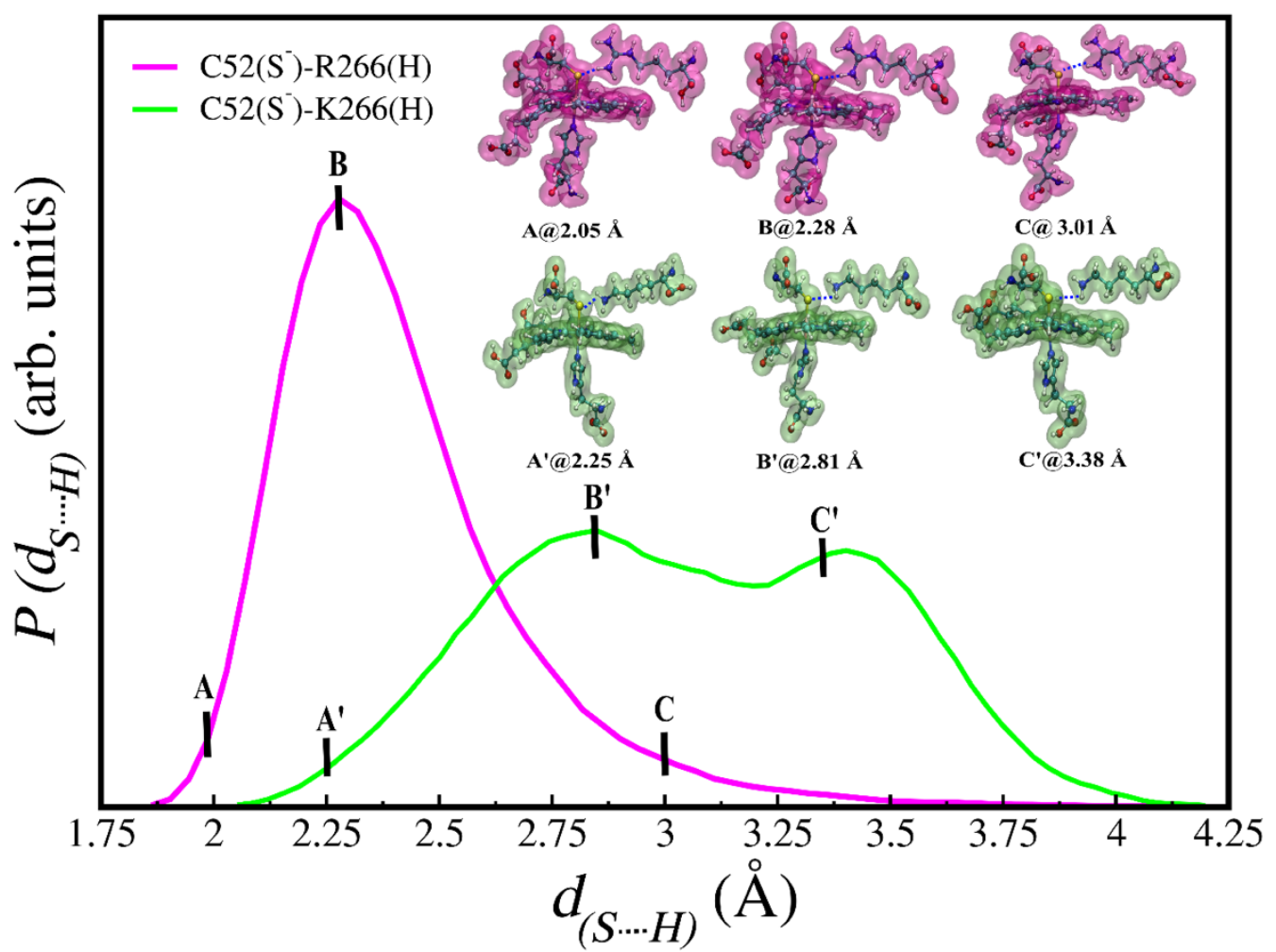

Figure 5. The electron density obtained as computed employing DFT (PBE0) calculations (isosurface value $0.025 \mathrm{e}^{-/} \AA^{3}$ ) at the configurations with the selected distances as indicated in the probability distribution curve of hydrogen-bonding between Cys52 -- Arg/Lys266. The selected H-bonding distances for Arg266 with Cys52 are



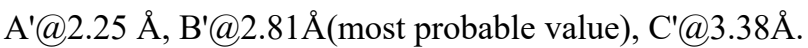

\subsection{Role of Asp316, Alpha helix 8 and PLP Binding sites in the bidirectional}

\section{Communications}

It is now quite evident that the long-distance interactions between the Cys52(S-)-Arg266(H) are the key contributor to the bi-directional electronic communications between the Heme and PLP catalytic center. Breaking of this interaction causes loss of enzymatic activities. Furthermore, the analysis of the MD trajectories revealed that Asp316 residue plays a pivotal role in such long-distant interactions as well by providing a favorable environment for the 
Arg266, that manifests a stable and strong non-covalent hydrogen-bonding with the Cys52( $\mathrm{S}^{-}$ ). The de-protonated anionic oxygen in the side chain of Asp316 forms a strong hydrogen bonding with the neighboring residue especially with the Arg266 in CBS ${ }^{\mathrm{WT}}$ and Lys266 in $\mathrm{CBS}^{\mathrm{R} 266 \mathrm{~K}}$. On the other hand, $\alpha$-helix 8 is a common communicator between Heme and the PLP site which contains Arg266/Lys266 of the protein. Thus, it is a part of the communication pathway between heme and the PLP site. The comparison of the RMSD between CBS ${ }^{\mathrm{WT}}$ and $\mathrm{CBS}^{\mathrm{R} 266 \mathrm{~K}}$ (SI section 3.4) reveals that Alpha helix 8 in $\mathrm{CBS}^{\mathrm{WT}}$ protein is slightly more compact compared to the mutated case. The R266K variant has unique features as compared to other mutations as it is enzymatically competent with CBS wild-type. It has been reported that R266K exhibits different enzymatic activity for different substrates ${ }^{22,23}$. The question is still there if there is any significant local structural and dynamical impact on the catalytic PLPbinding domain that might be impacting the enzymatic activities? Comparing the PLP binding sites between the WT and mutated case it is quite evident that there is no significant change is observed between the PLP and corresponding bindings sites of the enzyme. The pattern of the depicted interactions shown in Figure 6 is comparable between the WT and R266K hCBS across the trajectories.

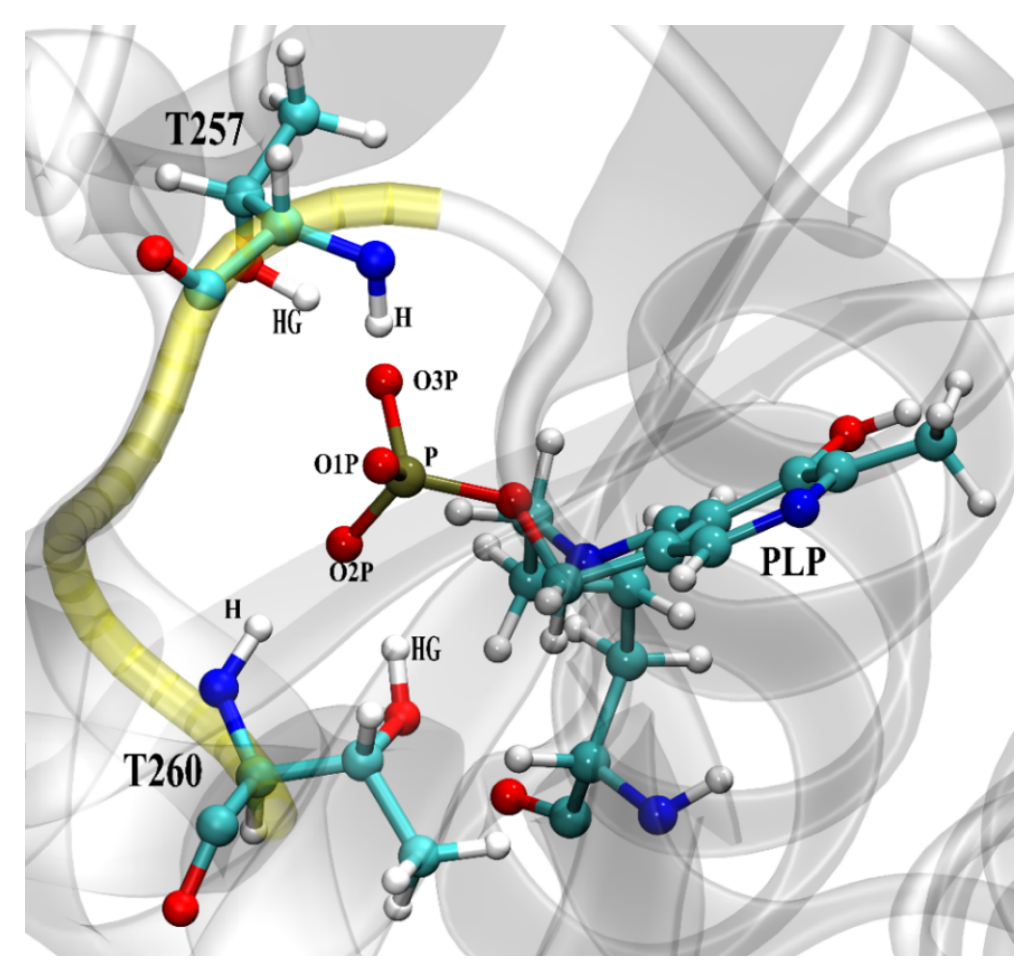

Figure 6. PLP phosphate group interaction with the allosteric residue T257 and T260. 


\section{Conclusions}

We have compared the structural dynamics of the hCBS enzyme employing the classical molecular dynamics (MD) simulations for wild-type (hCBS ${ }^{\mathrm{WT}}$ ) and the mutated $\left(\mathrm{hCBS}^{\mathrm{R} 266 \mathrm{~K}}\right)$ one. The hCBS contains a Heme cofactor, which is attached to the protein through the axial coordination bonding with one cysteine and one histidine. The hexacoordinated Heme is in the low spin-state (singlet) and for which the forcefield parameters are explicitly developed and benchmarked with the various density functional theory-based static calculations. Our simulations captured the long-range allosteric interactions between the Heme and the catalytic PLP center and confirm the previous experimental observations. In this work, we have realized that the Arg266 interacts strongly with the Heme co-factor through a strong hydrogen bond with Cys52 residue. The singular peak in the probability distribution curve of this particular hydrogen bonding confirms the stable and strong hydrogen-bond formation with a rigid structural motif of the Arg266 due to hydrogen-bonds with Cys52 as well as with Asp316. The R266K mutation invokes disruptions to this stable structural motif and also reduce the overlap of the electronic states. Hence, this is the primary reason behind the decrease of allosteric communications between the Heme and the catalytic PLP center due to the R266K mutations that reduces the enzymatic activities. The R266K mutations not only break the strong hydrogen-bonding pattern but also modulate the electronic communications. In the longdistance interactions between the heme and catalytic center, the Cys52( $\left.\mathrm{S}^{-}\right)-\mathrm{Arg} 266(\mathrm{H})$ the noncovalent hydrogen bonding plays the key role, and breaking of this interaction causes the loss of enzymatic activities.

\section{Acknowledgments}

The authors thank Aritra Mukhopadhyaya and Rehan Khan for various helpful discussions. We acknowledge the financial support from the Department of Science and Technology through SERB-ECR project no. ECR/2016/000362 and Indo-Sweden joint project no. DST/INT/SWD/VR/P-01/2016. The Ph.D. fellowship support by the Institute of Nano Science and Technology (INST) is thankfully acknowledged.

\section{Supporting Information}

Details of Heme force field parameter generation and benchmarking, PLP force field preparation, structural parameters comparison for $\mathrm{CBS}^{\mathrm{WT}}$ and $\mathrm{CBS}^{\mathrm{R} 266 \mathrm{~K}}$. 


\section{References}

1. Fowler, B., Disorders of homocysteine metabolism. J. Inherit. Metab. Dis. 1997, 20, 270-85.

2. de Koning, A.B.; Werstuck, G.H.; Zhou, J.; Austin, R.C., Hyperhomocysteinemia and its role in the development of atherosclerosis. Clin. Biochem. 2003, 36, 431-41.

3. Majtan, T.; Pey, A.L.; Ereño-Orbea, J.; Martínez-Cruz, L.A.; Kraus, J.P., Targeting Cystathionine Beta-Synthase Misfolding in Homocystinuria by Small Ligands: State of the Art and Future Directions. Curr. Drug. Targets. 2016, 17, 1455-70.

4. Singh, S.; Padovani, D.; Leslie, R.A.; Chiku, T.; Banerjee, R., Relative Contributions of Cystathionine $\beta$-Synthase and $\gamma$-Cystathionase to $\mathrm{H}_{2} \mathrm{~S}$ Biogenesis via Alternative Trans-sulfuration Reactions. J. Biol. Chem. 2009, 284, 22457-66.

5. Braunstein, A.E.; Goryachenkova, E.V.; Tolosa, E.A.; Willhardt, I.H.; Yefremova, L.L., Specificity and some other properties of liver serine sulphhydrase: Evidence for its identity with cystathionine $\beta$-synthase. Biochimica et Biophysica Acta. 1971, 242, 247-260.

6. Papadopoulos, A.I.; Walker, J.; Barrett, J., A novel cystathionine Beta-synthase from Panagrellus redivivus (Nematoda). Int J Biochem Cell Biol. 1996, 28, 543-549.

7. Walker, J.; Barrett, J.; Thong, K.W., The identification of a variant form of cystathionine Beta-synthase in nematodes. Exp. Parasitol. 1992, 75, 415-424.

8. Majtan, T.; Krijt, J.; Sokolová, J.; Křížková.; M.; Ralat. M.A.; Kent. J.; Gregory, J.F.; Kožich, V.; Kraus. J.P., Biogenesis of Hydrogen Sulfide and Thioethers by Cystathionine Beta-Synthase. Antioxid. Redox. Signal. 2018, 28, 311-323.

9. Taoka, S.; West, M.; Banerjee, R., Characterization of the Heme and Pyridoxal Phosphate Cofactors of Human Cystathionine $\beta$-Synthase Reveals Nonequivalent Active Sites. Biochemistry. 2009, 38, 2738-2744.

10. Meier, M.; Janosik, M.; Kery, V.; Kraus, J.P.; Burkhard, P. Structure of human cystathionine beta-synthase: a unique pyridoxal 5'-phosphate-dependent heme protein. EMBO. J. 2001, 20, 3910-6.

11. Ereño-Orbea, J.; Majtan, T.; Oyenarte, I.; Kraus, J.P.; Martínez-Cruz, L.A., Structural basis of regulation and oligomerization of human cystathionine $\beta$-synthase, the central enzyme of transsulfuration. Proc. Natl. Acad. Sci. U. S. A. 2013, 110, E3790-9.

12. Ereño-Orbea, J.; Majtan, T.; Oyenarte, I.; Kraus, J.P.; Martínez-Cruz, L.A.Structural insight into the molecular mechanism of allosteric activation of human cystathionine $\beta$ - 
synthase by S-adenosylmethionine. Proc. Natl. Acad. Sci. U. S. A. 2014, 111, E384552.

13. Taoka, S.; Ohja, S.; Shan, X.; Kruger, W.D.; Banerjee, R., Evidence for heme mediated redox regulation of human cystathionine beta-synthase activity. J. Biol. Chem. 1998, $273,25179-84$.

14. Majtan, T.; Singh, L.R.; Wang, L.; Kruger, W.D.; Kraus, J.P., Active Cystathionine $\beta$ Synthase Can Be Expressed in Heme-free Systems in the Presence of Metal-substituted Porphyrins or a Chemical Chaperone. J. Biol. Chem. 2008, 283, 34588-34595.

15. Oliveriusová, J.; Kery, V.; Maclean, K.N.; Kraus, J.P., Deletion mutagenesis of human cystathionine beta-synthase. Impact on activity, oligomeric status, and Sadenosylmethionine regulation. J. Biol. Chem. 2002, 277, 48386-94.

16. Kery, V.; Poneleit, L.; Meyer, J.D.; Manning, M.C.; Kraus, J.P., Binding of pyridoxal 5'-phosphate to the heme protein human cystathionine beta-synthase. Biochemistry. 1999, 38, 2716-24.

17. Singh, S.; Madzelan, P.; Stasser, J.; Weeks, C.L.; Becker, D.; Spiro, T.G.; Penner-Hahn, J.; Banerjee, R., Modulation of the heme electronic structure and cystathionine $\beta$ synthase activity by second coordination sphere ligands: The role of heme ligand switching in redox regulation. J. Inorg. Biochem. 2009, 103, 689-697.

18. Gherasim, C.; Yadav, P.K.; Kabil, O.; Niu, W.; Banerjee, R., Nitrite Reductase Activity and Inhibition of $\mathrm{H}_{2} \mathrm{~S}$ Biogenesis by Human Cystathionine $\beta$-Synthase. Plos. One. 2014, 9, e85544.

19. Vicente, J.B.; Colac, H.G.; Mendes, M.I.S.; Sarti, P.; Leandro, P.; Giuffre, A., NO* Binds Human Cystathionine ß-Synthase Quickly and Tightly. J. Biol. Chem. 2014, 289, 8579-87.

20. Singh, S.; Madzelan, P.; Banerjee, R., Properties of an unusual heme cofactor in PLPdependent cystathionine $\beta$-synthase. Nat. Prod. Rep. 2007, 24, 631-639.

21. Weeks, C.L.; Singh, S.; Madzelan, P.; Banerjee, R.; Spiro, T.G., Heme regulation of human cystathionine $\beta$-synthase activity: Insights from fluorescence and Raman spectroscopy. J. Am. Chem. Soc. 2009, 131, 12809-12816.

22. Smith, A.T.; Su, Y.; Stevens, D.J.; Majtan, T.; Kraus, J.P.; Burstyn, J.N., Effect of the Disease-Causing R266K Mutation on the Heme and PLP Environments of Human Cystathionine $\beta$-Synthase. Biochemistry. 2012, 51, 6360-6370. 
23. Majtan, T.; Kraus, J.P., Folding and activity of mutant cystathionine $\beta$-synthase depends on the position and nature of the purification tag: characterization of the R266K CBS mutant. Protein Expr Purif. 2012, 82, 317-24.

24. Yadav, P.K.; Xie, P.; Banerjee, R., Allosteric communication between the PLP and heme sites in the $\mathrm{H}_{2} \mathrm{~S}$-generator human cystathionine $\beta$-synthase. J. Biol. Chem. 2012, $287,37611-37620$.

25. Cornell, W.D.; Cieplak, P.; Bayly, C.I.; Gould, I,R.; Merz, K.M.; Ferguson, D.M.; Spellmeyer, D.C.; Fox, T.; Caldwell, J.W.; Kollman, P.A., A Second-Generation Force Field for the Simulation of Proteins, Nucleic Acids, and Organic Molecules. J. Am. Chem. Soc. 1995, 117, 5179-5197.

26. MacKerell Jr, A. D.; Bashford, D.; Bellott, M.; Dunbrack Jr, R. L.; Evanseck, J. D.;Field, M. J.; Fischer, S.; Gao, J.; Guo, H.; Ha, S. et al. All-atom empirical potential for molecular modeling and dynamics studies of proteins. J. Phys. Chem. B. 1998, 102, 3586-3616.

27. Autenrieth, F.; Tajkhorshid, E.; Baudry, J.; Luthey-Schulten, Z., Classical force field parameters for the heme prosthetic group of cytochrome c. J. Comput. Chem. 2004, 25, 1613-22.

28. Oda, A.; Yamaotsu, N.; Hirono, S., New AMBER Force Field Parameters of Heme Iron for Cytochrome P450s Determined by Quantum Chemical Calculations of Simplified Models. J. Comput. Chem. 2005, 26, 818-826.

29. Chillemi, G.; Anselmi., M.; Sanna, N.; Padrin, C.; Balducci, L.; Cammarata, M.; Pace, E.; Chergui, M.; Benfatto., M., Dynamic multiple-scattering treatment of X-ray absorption: Parameterization of a new molecular dynamics force field for myoglobin. Struct. Dyn. 2018, 5, 054101.

30. Doss, C.G.P.; Rajith, B.; Magesh, R.; Kumar, A.A., Influence of the SNPs on the structural stability of CBS protein: Insight from molecular dynamics simulations. Front. Bio. 2014, 9, 505-518.

31. Gupta, S.; Kelow, S.; Wang, L.; Andrake, M.D.; Roland L. Dunbrack Jr, R.L.; Kruger, W.D.,Mouse modeling and structural analysis of the p.G307S mutation in human cystathionine $\beta$-synthase $(C B S)$ reveal effects on CBS activity but not stability. J. Biol. Chem. 2018, 293, 13921-13931. 
32. Pettersen, E.F.; Goddard, T.D.; Huang, C.C.; Couch, G.S.; Greenblatt, D.M.; Meng, E.C.; Ferrin, T.E., UCSF Chimera--a visualization system for exploratory research and analysis. J. Comput. Chem. 2004, 25, 1605-12.

33. Dolinsky, T.J.; Czodrowski, P.; Li, H.; Nielsen, J.E.; Jensen, J.H.; Klebe, G.; Baker, N.A., PDB2PQR: expanding and upgrading automated preparation of biomolecular structures for molecular simulations. Nucleic. Acids. Res. 2007, 35, W522-525.

34. Li, P.; Merz, K.M. MCPB.py: A Python Based Metal Center Parameter Builder. $J$. Chem. Inf. Model. 2016, 56, 599-604.

35. Mark. P.; Nilsson, L., Structure and Dynamics of the TIP3P, SPC, and SPC/E Water Models at 298 K. J. Phys. Chem. 2001, 105, 9954-9960.

36. Case, D. A.; Cheatham, T. E.; Darden, T.; Gohlke H.; Luo, R.; Merz, K. M.; Onufriev, A.; Simmerling, C.; Wang, B.; and Woods. R. J., The Amber biomolecular simulation programs. J. Computat. Chem. 2005, 26, 1668-1688.

37. Maier, J.A.; Martinez, C.; Kasavajhala, K.; Wickstrom, L.; Hauser, K.E; Simmerling, C., ff14SB: Improving the Accuracy of Protein Side Chain and Backbone Parameters from ff99SB. J. Chem. Theory. Comput. 2015, 11, 3696-713.

38. Darden, T.; York, D.; Pedersen, L., Particle mesh Ewald: An $N \cdot \log (N)$ method for Ewald sums in large systems. J. Chem. Phys. 1993, 98, 10089.

39. Phillips, J. C.; Braun, R.; Wang, W.; Gumbart, J.; Tajkhorshid, E.; Villa, E.; Chipot, C.; Skeel, R. D.; Kalé, L.; and Schulten, K., Scalable molecular dynamics with NAMD. J. Comput. Chem. 2005. 26, 1781-1802

40. Humphrey, W.; Dalke, A.; and Schulten, K., VMD-Visual Molecular Dynamics. $J$. Molec. Graphics, 1996, 14, 33-38.

41. Grant, B.J.; Rodrigues, A.P.C.; El Sawy, K.M., McCammon, J.A.; Caves, L.S.D., Bio3D: An $\mathrm{R}$ package for the comparative analysis of protein structures. Bioinformatics. 2006, 22, 2695-2696. 